\title{
Stability and nonlinear regimes of flow over a saturated porous medium
}

\author{
T. P. Lyubimova ${ }^{1,2}$, D. T. Baydina ${ }^{2}$, and D. V. Lyubimov ${ }^{2}$ \\ ${ }^{1}$ Institute of Continuous Media Mechanics, Ural Branch of RAS, Perm, Russia \\ ${ }^{2}$ Perm State University, Theoretical Physics Department, Perm, Russia \\ Correspondence to: T. P. Lyubimova (lubimova@psu.ru)
}

Received: 1 January 2013 - Revised: 12 June 2013 - Accepted: 14 June 2013 - Published: 23 July 2013

\begin{abstract}
The paper deals with the investigation of stability and nonlinear regimes of flow over the saturated porous medium applied to the problem of stability of water flow over the bottom covered with vegetation. It is shown that the velocity profile of steady plane-parallel flow has two inflection points, which results in instability of this flow. The neutral stability curves, the dependencies of critical Reynolds number and the wave number of most dangerous perturbations on the ratio of porous layer thickness to the total thickness are obtained. The nonlinear flow regimes are investigated numerically by finite difference method. It is found that at supercritical parameter values waves travelling in the direction of the base flow take place.
\end{abstract}

\section{Introduction}

Investigation of the stability of flows over a porous medium saturated with liquid is important to understand the processes associated with the propagation of wind in areas of vegetation and over vegetation canopy, as well as the stability of water flows over the aquatic vegetation. In the propagation of the wind in the areas of vegetation, transfer of various physical quantities across the boundaries affects many atmospheric processes. Examples are the destruction of trees and shrubs by the wind, precipitation and propagation of dust and other suspensions.

The motion of air under the canopy of vegetation is substantially nonuniform; the main contribution is made by coherent structures, the characteristic size of which is comparable to the size of the canopy vegetation. It is now believed that the main characteristics of the flow of air near the canopy of vegetation are similar to the corresponding values of shear flow (Raupach et al., 1996). Due to the friction of the vegetation, the average velocity of airflow under the canopy is less than the velocity above the canopy, and the typical vertical profile of the average velocity has an inflection point. The sharp drop of tangential velocity component promotes the development of instability similar to the Kelvin-Helmholtz instability. The coherent structures arising at the boundary of vegetation canopy due to the Kelvin-Helmholtz instability are travelling waves of horizontal transverse rolls rotating in one direction and propagating with the characteristic velocity equal to the flow velocity at the inflection point.

The structures described above are observed not only in atmospheric flows, but also in water systems (Ghisalberti and Nepf, 2002). Stability of Poiseuille flow in a fluid layer overlying highly porous layer was studied in Chang et al. (2006), Hill and Straughan (2008), and White and Nepf (2007). In the present paper we investigate the stability and nonlinear regimes of flow over the porous medium applied to the problem of stability of water flow over the bottom, covered with vegetation. This study is important due to the applications to the problem of a removal of contaminants from the bottom vegetation layer.

\section{Problem formulation}

Let us consider a two-layer system consisting of a layer of a viscous incompressible fluid and a porous layer saturated with the same fluid located underneath (Fig. 1). The lower boundary is assumed to be rigid, while the upper boundary is free and non-deformable. The layers are inclined to the horizontal position which corresponds to the river slope. The question on the boundary conditions at the interface of fluid layer and fluid-saturated porous layer is still under 


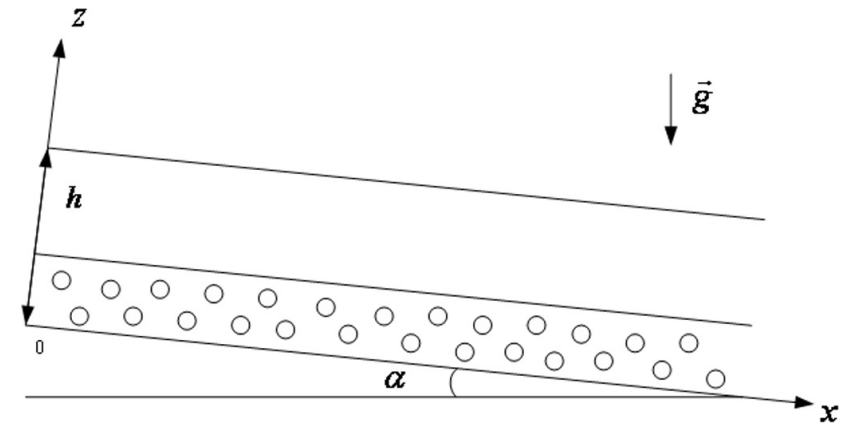

Fig. 1. Problem configuration.

discussion, which is why we describe the fluid motion in a two-layer system on the basis of a so-called one-equation model (see, for example, Chen and Chen, 1988). According to this approach, we use the equations for fluid-saturated porous medium in both layers, and the presence of two different layers is modelled by introducing the non-uniform (depending on the coordinate across the layer) permeability and porosity.

Description of the fluid-saturated porous medium is performed in the framework of the Brinkman model, in terms of the average velocity of the fluid in the pores:

$\rho\left[\frac{\partial \boldsymbol{V}}{\partial t}+(\boldsymbol{V} \nabla) \boldsymbol{V}\right]=-\nabla p-\frac{\mu}{K} \varphi \boldsymbol{V}+\tilde{\mu} \Delta \boldsymbol{V}+\rho \boldsymbol{g}$,

$\operatorname{div}(\varphi \boldsymbol{V})=0$.

Here $\boldsymbol{V}$ is the average flow velocity in pores, $\rho$ the fluid density, $\mu$ the dynamic viscosity, $\tilde{\mu}$ the effective viscosity, and $\varphi$ and $K$ the porosity and permeability of porous medium; it is assumed that they vary across the layer, and $\boldsymbol{g}$ is the gravity acceleration; the effective viscosity $\tilde{\mu}$ is accepted to be equal to $\mu$.

Let us choose the following scales: full thickness $h$ for the length, characteristic velocity $U=\left(g h^{2} \sin \alpha\right) / v$ for the velocity, double maximal velocity of the run-off of a homogeneous fluid layer on an inclined plane for the time $h^{2} / \nu$, and $\rho g h \sin \alpha$ for the pressure, where $v=\mu / \rho$ is the kinematic viscosity, and $\alpha$ is the angle of layer inclination to the horizontal position.

The equations in the dimensionless form are

$\frac{\partial \boldsymbol{V}}{\partial t}+\operatorname{Re}(\boldsymbol{V} \nabla) \boldsymbol{V}=-\nabla p-D a^{-1} \frac{\varphi}{\kappa} \boldsymbol{V}+\Delta \boldsymbol{V}-(\sin \alpha)^{-1} \boldsymbol{e}_{\mathrm{V}}$,

$\operatorname{div}(\varphi \boldsymbol{V})=0$.

Here $D a=K_{0} / h^{2}$ is the Darcy number, $R e=U h / v$ the Reynolds number, $\kappa=K / K_{0}$ the dimensionless permeability of porous medium, $K_{0}$ the characteristic value of the permeability, and $\boldsymbol{e}_{\mathrm{v}}$ the unit vector directed vertically upward.

On the lower boundary of the system, the no-slip condition is imposed, and on the upper boundary the conditions of the normal component of velocity and shear stress vanish; the ambient pressure is taken as the reference value, with the direction of the coordinate axes shown in Fig. 1:

$z=0: \boldsymbol{V}=0$

$z=1: \boldsymbol{V}_{z}=0,\left(\frac{\partial \boldsymbol{V}_{x}}{\partial z}+\frac{\partial \boldsymbol{V}_{z}}{\partial x}\right)=0, p=0$.

\section{Steady flow}

We seek a solution for (2)-(3) corresponding to the steady plane-parallel flow $\boldsymbol{V}_{0}=\left(\boldsymbol{V}_{0}(z), 0,0\right)$. The equations describing such a solution, in the projections on the directions $x$ and $z$, are as follows:

$\frac{\partial p_{0}}{\partial x}=-D a^{-1} \frac{\varphi}{\kappa} \boldsymbol{V}_{0}+\frac{\partial^{2} \boldsymbol{V}_{0}}{\partial z^{2}}+1, \frac{\partial p_{0}}{\partial z}=-\operatorname{ctg} \alpha$.

The boundary conditions are

$z=0: \boldsymbol{V}_{0}=0 ; \quad z=1: \frac{\partial \boldsymbol{V}_{0}}{\partial z}=0, p=0$.

Integrating the second equation in (4) with respect to $z$, we obtain $p_{0}=-z \operatorname{ctg} \alpha+C(x)$. Accounting for the boundary condition for the pressure at the free surface $p_{0}(1)=0$ gives $C(x)=\operatorname{ctg} \alpha$. Finally, for $p_{0}$ we obtain $p_{0}=(1-z) \operatorname{ctg} \alpha$, from which we have $\partial p_{0} / \partial x=0$. For $\boldsymbol{V}_{0}$ we obtain the equation

$-D a^{-1} \frac{\varphi}{\kappa} \boldsymbol{V}_{0}+\frac{\partial^{2} \boldsymbol{V}_{0}}{\partial z^{2}}+1=0$

with the boundary conditions

$z=0: \boldsymbol{V}_{0}=0 ; \quad z=1: \frac{\partial \boldsymbol{V}_{0}}{\partial z}=0$.

For the function $\varphi / \kappa$, we introduce the following approximation:

$\frac{\varphi}{\kappa}=\frac{(d-z)}{\sqrt{1+a(d-z)^{2}}}-\frac{(d-1)}{\sqrt{1+a(d-1)^{2}}}$.

Equation (8) contains two parameters: parameter $a$, which defines the thickness of the transient layer between the pure fluid and fluid-saturated porous medium, and parameter $d$, which is the ratio of porous layer thickness to the total thickness. The calculations were performed for different values of these parameters. In Fig. 2 the function $\varphi / \kappa$ is plotted for $a=100, d=0.5$.

Equations (6)-(7) were solved numerically by finite difference method. In Fig. 3 the velocity profile for steady flow is plotted for $a=100, d=0.5, D a=0.0001$. The profile contains two inflection points: one in the liquid layer, another in the porous medium. The presence of inflection points in the velocity profile can lead to the instability of steady flow. 


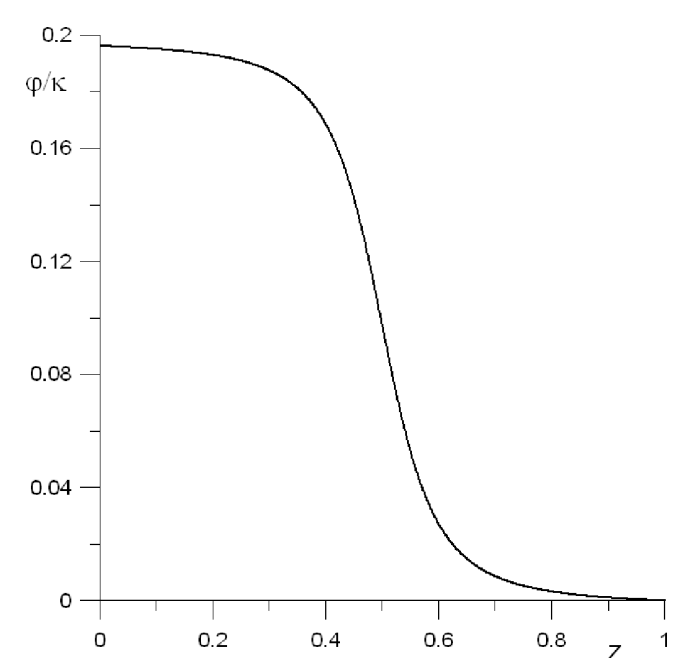

Fig. 2. Distribution of $\varphi / \kappa$ across the layer for $a=100, d=0.5$.

\section{Linear stability of steady flow}

Consider now the stability of steady flow with respect to small perturbations. Linearized equations for small perturbations read as follows:

$$
\begin{aligned}
& \frac{\partial \boldsymbol{V}^{\prime}}{\partial t}+\operatorname{Re}\left(\boldsymbol{V}_{0} \cdot \nabla \boldsymbol{V}^{\prime}+\boldsymbol{V}^{\prime} \cdot \nabla \boldsymbol{V}_{0}\right)=-\nabla p^{\prime}-D a^{-1} \frac{\varphi}{\kappa} \boldsymbol{V}^{\prime} \\
& +\Delta \boldsymbol{V}^{\prime}, \operatorname{div}\left(\varphi \boldsymbol{V}^{\prime}\right)=0 .
\end{aligned}
$$

We restrict ourselves to the plane perturbations $V^{\prime}=$ $(u, 0, w)$. Eliminating the pressure and horizontal velocity components from the equations and introducing the vorticity $\omega=\operatorname{curl}_{y} V^{\prime}$, we obtain

$$
\begin{gathered}
\frac{\partial \omega}{\partial t}+\operatorname{Re}\left(\boldsymbol{V}_{0} \frac{\partial \omega}{\partial x}+w \boldsymbol{V}_{0}^{\prime \prime}-\frac{\varphi^{\prime}}{\varphi} \boldsymbol{V}_{0}^{\prime} w\right) \\
=\Delta \omega-D a^{-1}\left[\frac{\varphi}{\kappa} \omega+\left(\frac{\varphi}{\kappa}\right)^{\prime} u\right], \\
\frac{\partial u}{\partial x}+\frac{\partial w}{\partial z}+\frac{\varphi^{\prime}}{\varphi} w=0 .
\end{gathered}
$$

We consider the perturbations periodical in $x$ direction:

$w=W(z, t) \exp (i k x), u=U(z, t) \exp (i k x)$,

$\omega=\Omega(z, t) \exp (i k x)$.

Substituting the perturbations in this form into the equations and introducing new variable $\bar{\Omega} \equiv-i k \Omega$, we obtain

$$
\begin{gathered}
\frac{\partial \bar{\Omega}}{\partial t}+i k \operatorname{Re}\left(\boldsymbol{V}_{0} \bar{\Omega}-\boldsymbol{V}^{\prime \prime}{ }_{0} W+\frac{\varphi^{\prime}}{\varphi} \boldsymbol{V}_{0}^{\prime} W\right)=\left(\bar{\Omega}^{\prime \prime}-k^{2} \bar{\Omega}\right) \\
-D a^{-1}\left[\frac{\varphi}{\kappa} \bar{\Omega}+\left(\frac{\varphi}{\kappa}\right)^{\prime}\left(W^{\prime}+\frac{\varphi^{\prime}}{\varphi} W\right)\right]
\end{gathered}
$$

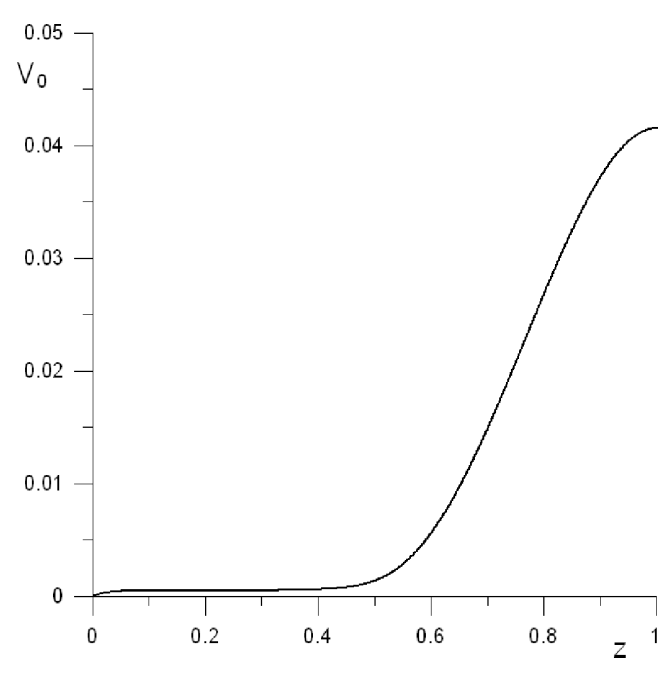

Fig. 3. Velocity profile for steady flow at $a=100, d=0.5$, $D a=0.0001$.

$W^{\prime \prime}-k^{2} W+\left(\frac{\varphi^{\prime}}{\varphi} W\right)^{\prime}=\bar{\Omega}$

$z=0: W=W^{\prime}=0 ; \quad z=1: W=W^{\prime \prime}=0$.

The stability problem contains not only the combination of the porosity and permeability but also porosity itself. We used the following approximation for the function $\varphi(z)$ that describes the dependence of the porosity on the coordinate across the layer:

$\varphi(z)=\varphi_{0}+\left(1-\varphi_{0}\right) \tanh (b z) / \tanh (b)$.

In this expression $\varphi_{0}$ is the value of porosity at $z=0$, while the parameter $b$ determines the thickness of the transition layer between the layers of the fluid and the porous medium. Main calculations were performed at $\varphi_{0}=0.1, b=5$. In Fig. 4 the function $\varphi(z)$ is plotted for these values of the parameters.

Equations (11)-(13), which define the temporal evolution of small perturbations of the base flow, were solved numerically by finite difference method. Equation (12) was solved at each time step by the scalar sweep method. Main calculations were carried out using a uniform grid with the number of nodes equal to 100 . The calculations for each parameter set were performed as long as the temporal evolution of the perturbation does not begin to be defined only by the most dangerous mode.

In Fig. 5 the neutral stability curves of base flow are plotted for different ratios $d$ of porous layer thickness to the total thickness. As one can see, at $d>0.5$ with an increase in the thickness of the porous layer (i.e. with the decrease of liquid layer thickness), the instability threshold grows. This is due 


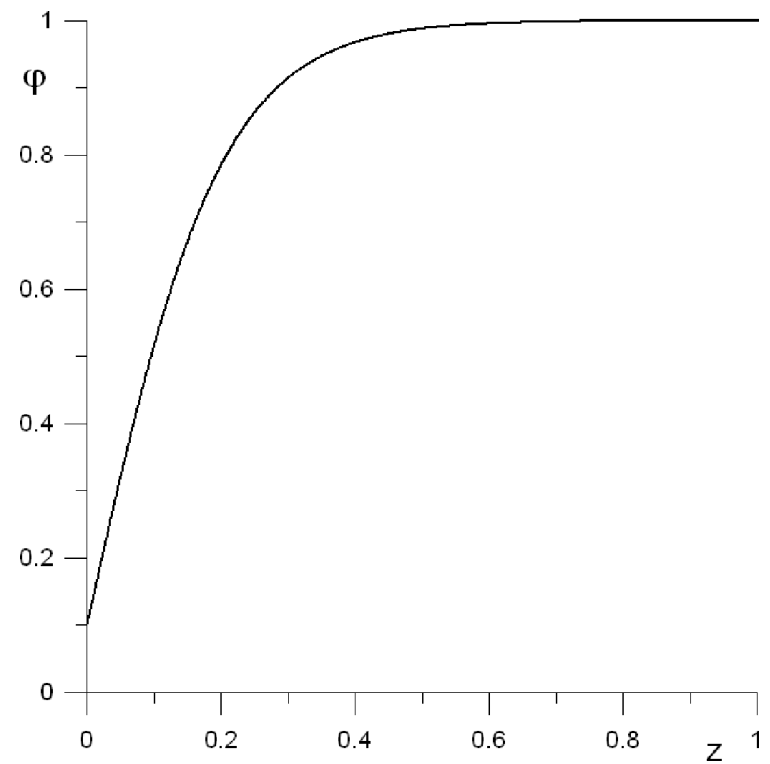

Fig. 4. Porosity distribution across the layer for $\varphi_{0}=0.1, b=5$.

to the fact that the instability is determined primarily by the perturbations developing in the liquid layer.

The frequency of critical perturbations is non-zero, so, as for conventional Kelvin-Helmholtz instability, the instability is of the oscillatory type.

\section{Nonlinear flow regimes}

We also studied the nonlinear flow regimes. The twodimensional flows were considered. In this case it is convenient to introduce the stream function and vorticity. Since the divergence of the filtration velocity $\boldsymbol{V}$ is zero, and the divergence of the average velocity in the pores $\boldsymbol{V}$ is non-zero, the stream function $\psi$ was introduced for the filtration velocity: $v_{x}=\partial \psi / \partial z, v_{z}=-\partial \psi / \partial x$. However, since the full nonlinear equations in terms of the stream function and vorticity of the filtration velocity are quite complex due to the variability of the porosity and permeability, the calculations were based on the equations for the average velocity in the pores having a simpler form than the equations for the filtration velocity. The average velocity of the fluid in the pores $\boldsymbol{V}$ is related to the stream function $\psi$ for the filtration velocity as

$\boldsymbol{V}=\varphi^{-1} \boldsymbol{V}=\left(\varphi^{-1} \frac{\partial \psi}{\partial z},-\varphi^{-1} \frac{\partial \psi}{\partial x}\right)$

Vorticity $\Phi$ of the average velocity in the pores is $\Phi=$ $\operatorname{curl}_{y} \boldsymbol{V}$, from which we have the Poisson equation for the stream function $\psi$ :

$\Delta \psi-\frac{\varphi^{\prime}}{\varphi} \frac{\partial \psi}{\partial z}-\varphi \Phi=0$.

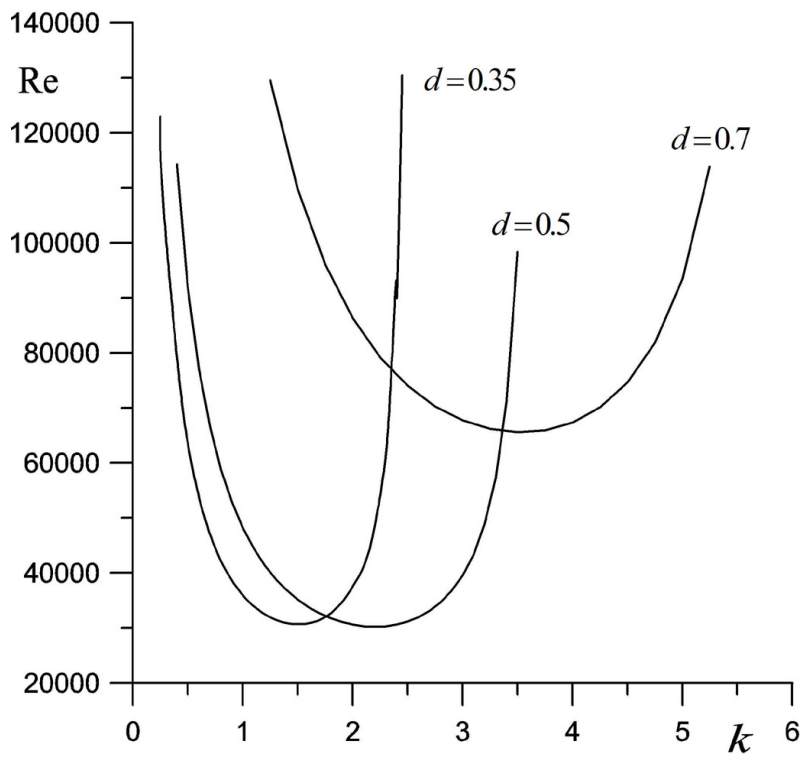

Fig. 5. Neutral curves for $D a=0.0001$ and different $d$.

Applying the operation $\operatorname{curl}_{y}$ to Eq. (2), we obtain

$$
\begin{gathered}
\frac{\partial \Phi}{\partial t}+\frac{1}{\varphi}\left(\frac{\partial \psi}{\partial z} \frac{\partial \Phi}{\partial x}-\frac{\partial \psi}{\partial x} \frac{\partial \Phi}{\partial z}\right)-\frac{\varphi^{\prime}}{\varphi^{2}} \Phi \frac{\partial \psi}{\partial x} \\
=\Delta \Phi-D a^{-1}\left(\frac{1}{\kappa} \Delta \psi-\frac{\kappa^{\prime}}{\kappa^{2}} \frac{\partial \psi}{\partial z}\right) .
\end{gathered}
$$

The boundary conditions on the rigid and free boundaries are as follows:

$z=0: \psi=0, \frac{\partial \psi}{\partial z}=0 ; \quad z=1: \psi=Q, \frac{\partial \psi}{\partial z}=0$,

where $Q=\int_{0}^{1} \varphi \boldsymbol{V} \mathrm{d} z$ is the flow rate. The calculations were performed under the assumption that the flow rate is not very different from the value corresponding to a steady flow, and the flow rate was assumed to be $Q=\int_{0}^{1} \varphi \boldsymbol{V}_{0} \mathrm{~d} z$.

Equations (14)-(16) were solved by finite difference method. The calculations were carried out for rectangular cavities with the length equal to the wavelength of the most dangerous perturbations according to the linear stability theory. On the lateral boundaries, the periodicity conditions were set.

The calculations show that at small values of the Reynolds number the transient process leads to the stationary regime: the stream function attains a constant value. Starting from a certain value of the Reynolds number, which depends on the ratio of the layer thicknesses and the Darcy number, the transient process leads to the regime of stationary oscillations with non-zero mean value. The amplitude and frequency of oscillations, as well as the average value around which the oscillations are performed, depend on the parameters. 

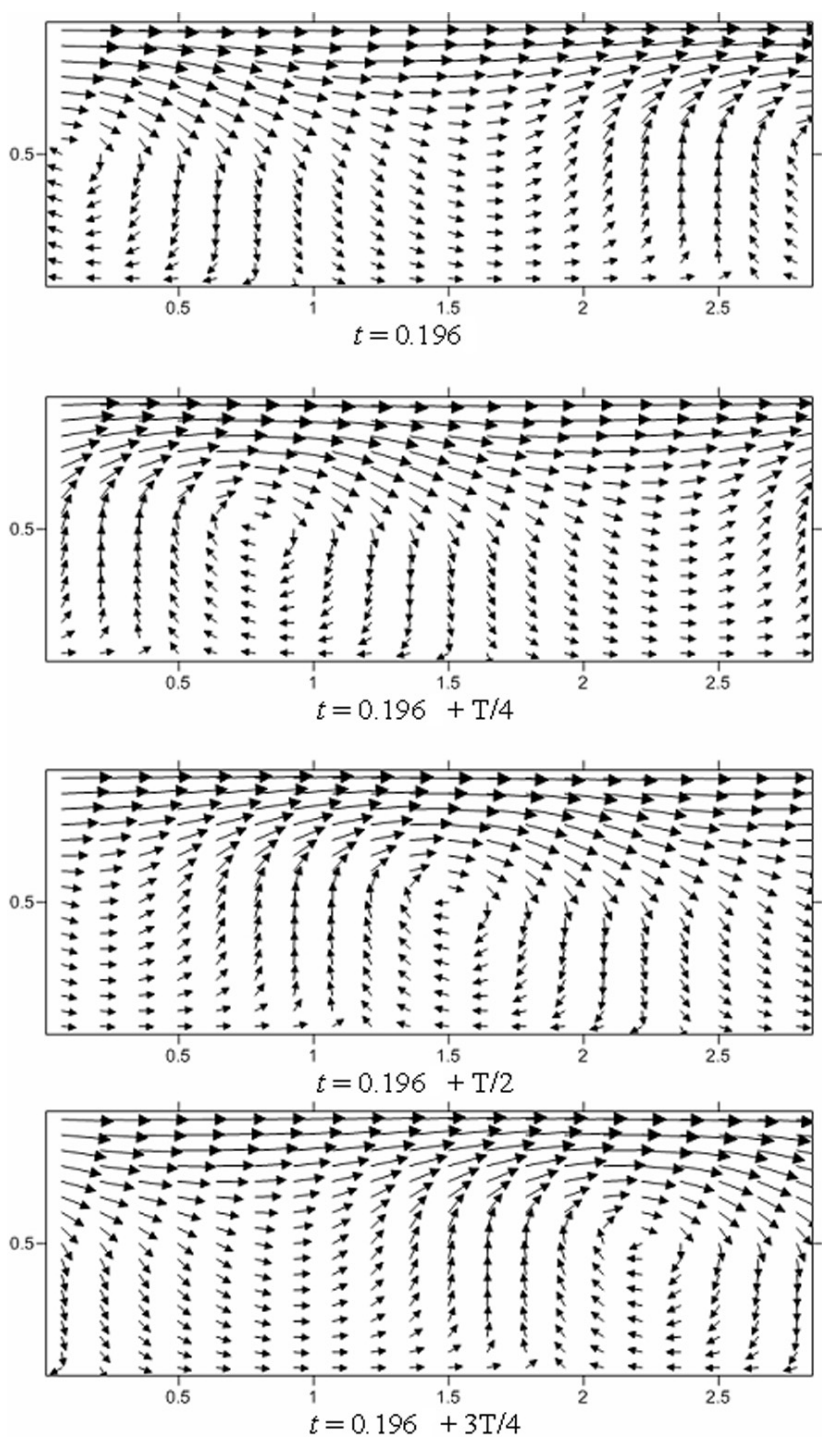

Fig. 6. Velocity vector field at $D a=0.0001, d=0.5, R e=40000$ and different phases of oscillation period.

The analysis of the dependencies of amplitude and frequency of oscillations on the Reynolds number for different values of the parameters $D a$ and $d$ shows that in all cases the bifurcation to the oscillatory regime is supercritical; close to the threshold, the oscillation amplitude $A$ changes with the growth of Reynolds number according to the square root law. Dependencies of the oscillation frequency on the Reynolds number for all values of the parameters $D a$ and $d$ covered by the calculations are close to linear. The critical Reynolds number and the frequency of oscillations in the threshold are close to the values obtained from linear stability analysis.

Figure 6 shows the instantaneous velocity vector fields for various phases of the oscillation period at fixed values of the parameters $D a=0.0001, d=0.5$, and $R e=40000$. As one can see, there are waves propagating in the direction of the base flow.

\section{Conclusions}

The flow over the porous medium has been investigated. It has been shown that the velocity profile of steady flow has two inflection points. The presence of inflection points results in instability of steady flow. The neutral stability curves, the dependencies of critical Reynolds number and wave number of the most dangerous perturbations on the ratio of porous layer thickness to the total thickness have been obtained. The nonlinear flow regimes have been investigated numerically by finite difference method. The instantaneous velocity fields are obtained for different Reynolds and Darcy numbers and ratios of layers thicknesses. It is found that at supercritical parameter values waves travelling in the direction of the base flow take place. Such a vortex flow may lead to the removal of contaminants from the bottom vegetation layer.

Acknowledgements. The work was carried out under financial support of Russian Foundation for Basic Research (grant 12-01-00795).

Edited by: R. Grimshaw

Reviewed by: two anonymous referees

\section{References}

Chang, M. H., Chen, F., and Straughan, B.: Instability of Poiseuille flow in a fluid overlying a porous layer, J. Fluid Mech., 564, 287303, doi:10.1017/S0022112006001583, 2006.

Chen, F. and Chen, C. F.: Onset of finger convection in a horizontal porous layer underlying a fluid layer, Heat Transf., 110, 403-409, doi:10.1115/1.3250499, 1988.

Ghisalberti, M. and Nepf, H. M.: Mixing layers and coherent structures in vegetated aquatic flows, J. Geophys. Res., 107, 1-11, doi:10.1029/2001JC000871, 2002.

Hill, A. A. and Straughan, B.: Poiseuille flow in a fluid overlying a porous medium, J. Fluid Mech., 603, 137-149, doi:10.1017/S0022112008000852, 2008.

Raupach, M. R., Finnigan, J. J., and Brunet, Y.: Coherent Eddies and Turbulence in Vegetation Canopies: The Mixing Layer Analogy, Bound.-Lay. Meteorol., 78, 351-382, 1996.

White, B. L. and Nepf, H. M.: Shear instability and coherent structures in shallow flow adjacent to a porous layer, J. Fluid Mech., 593, 1-32, 2007. 